\title{
Transport Processes in TlI and in the AgI-TlI-System
}

\author{
A. Schiraldi, A. Magistris, and E. Pezzati \\ Centro di studio per la termodinamica ed elettrochimica dei sistemi salini fusi e solidi del C.N.R. \\ c/o Istituto di Chimica-Fisica, Università di Pavia (Italy)
}

(Z. Naturforsch. 29 a, 782-785 [1974]; received March 7, 1974)

\begin{abstract}
The transport properties of $\mathrm{Tl}$ I and of the system $\mathrm{AgI}-\mathrm{Tl}$ I were investigated by measuring the electrical conductivity, $\sigma$, and the electronic and ionic transport numbers.

A particularly high electronic conductivity was detected in $\beta$-TlI, while the $\alpha$ phase showed a predominant anionic contribution, as in $\mathrm{TlCl}$ and $\mathrm{TlBr}$.

The intermediate compounds, $\mathrm{AgTl}_{2} \mathrm{I}_{3}$ and $\mathrm{AgTl}_{2}$, are silver ion conductors, but they exhibit low $\sigma$ values.

A comparison with other poliiodides, with a high silver ion conductivity, is suggested on the basis of the crystal bond ionicity.
\end{abstract}

A number of physico-chemical properties of $\mathrm{Tl}$ and Ag halides were already studied and compared by Tubandt and Lorenz ${ }^{1}$ and by Friauf ${ }^{2}$. The similarity observed between these two families of salts, noticeably pronounced in the case of the iodides (which also exhibit the common feature of allotropic modifications affectable by mechanical pressure $^{3-5}$ does not extend to the transport properties.

This is particularly true for $\mathrm{AgI}$ and TlI, as proved by the transport measurements reported in the present paper.

\section{Experimental}

A pellet obtained by pressing the yellow $\beta$-TII powder (Fluka p. a.; impurities $<20 \mathrm{ppm}$ ) showed a reddish surface colour corresponding to a partial $\beta \rightarrow \alpha$ transition and exhibited time dependent electrochemical properties; however, after two days annealing at $50^{\circ} \mathrm{C}$, it turned to the yellow colour and to the stable electrochemical properties of $\beta$-TII. Therefore specimens were always annealed before use.

The electrical conductivity was measured on pellets obtained by pressing TII powder between two silver powder layers. A recorder connected to an autobalancing Wayne Kerr bridge, mod. B 331, allowed to follow the signal change over the annealing period until stabilization and then to detect the conductivity behaviour with increasing temperature.

The electronic contribution was determined by suppressing the ionic part of the total electrical conductivity ${ }^{6,7}$ in a way quite similar to that employed by Vest and Tallan ${ }^{8}$ : the pellet was adjusted be-

Reprint requests to: Alberto Schiraldi, Istituto di Chimica-Fisica della Università di Pavia, Viale Taramelli, I-27100 Pavia (Italien). tween two graphite electrodes, to which a dc voltage (from an Amel mod. 551 potentiostat) lower than the decomposition potential of the salt, was established, while the current flow detected through a high sensitivity galvanometer Amel mod. 668 was recorded until a steady value, $\mathrm{I}_{\infty}$, was reached. Owing to the very low current signal an accurate screening of the experimental arrangement was required mainly for $\alpha$-TII specimens.

Measurements of the ionic transport numbers in TII, $t_{T l}$ and $t_{I}$, were carried out by the Tubandt method on a three pellets arrangement clasped between two silver discs.

\section{Results and Discussion}

\section{Transport Properties of TlI}

The conductivity data on TII samples were obtained either at stepwise stabilized temperatures or with a $2{ }^{\circ} \mathrm{C} / \mathrm{min}$ rising temperature. Both methods gave closely similar and reproducible results, fitted by the empirical equation

$$
\sigma=A \exp \{-B / T\}
$$

where $\sigma$ is the specific conductivity: for the $\alpha$ and $\beta$ phase regions, $A=1288$ and $0.992 \mathrm{ohm}^{-1} \mathrm{~cm}^{-1}$, $B=8600$ and $6500 \mathrm{~K}$ were respectively obtained.

Figure 1 shows the present results along with those by Phipps and Partridge ${ }^{9}$ and by Morlin ${ }^{10}$, the latter referring to single crystal samples. A satisfactory agreement is observed in the $\alpha$-phase region, where the relatively small deviations may be due to an uncertainty in the cell constant values and to differences in the contact resistances. On the contrary wider discrepancies between our and Morlin's results are evidenced in the $\beta$ phase region.

The $\beta \longleftrightarrow \alpha$ transition was recently proved to be not instantaneous by Samara ${ }^{5}$ and Morlin ${ }^{10}$, 


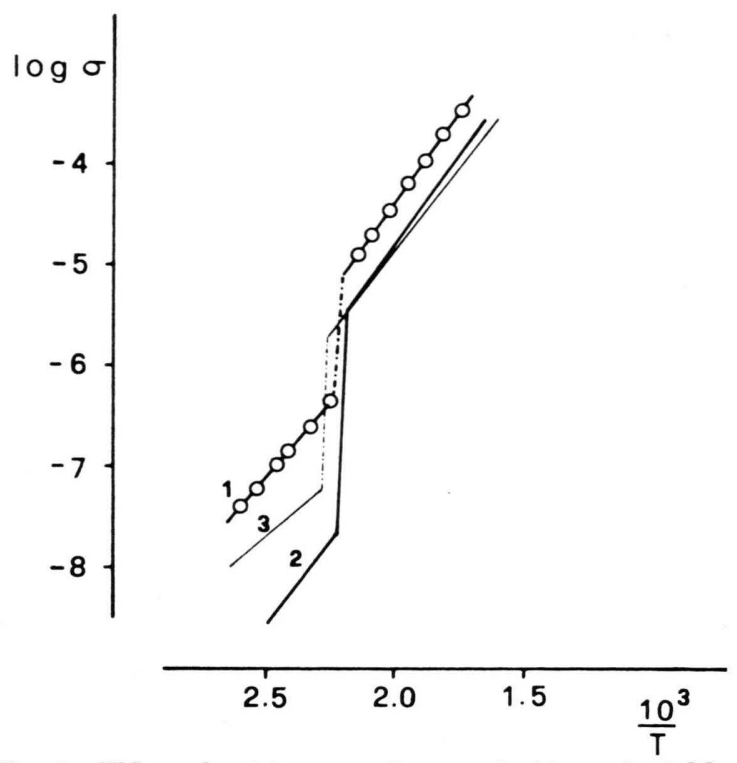

Fig. 1. Tl I conductivity according to: 1 this work, 2 Morlin, 3 Phipps and Patridge.

through dielectric constant measurements and TTT analysis respectively.

Our conductivity and DTA results allowed to state that the transition occurs at $182-185^{\circ} \mathrm{C}$ and that, whenever a TII sample undergoes a $\beta \longleftrightarrow \alpha$ transition, thermal annealing is compulsory in order to obtain a single phase specimen.

In fact it was possible to follow the transition process by observing the variation of the electronic contribution to the electrical conductivity, which is quite large for $\beta$-TII, but negligible for the $\alpha$ phase.

In a typical experiment, a $\beta$-TII sample showed at $160{ }^{\circ} \mathrm{C}$ a high and at $200{ }^{\circ} \mathrm{C}$ (after thermal stabilization) a negligible electronic conductivity: in the same sample, cooled down again to $160{ }^{\circ} \mathrm{C}$, the electronic conductivity was initially still low, but slowly reassumed, after some hours annealing, a constant value as large as at the beginning of the cycle.
By plotting $I_{\infty}$ vs $V$ (the latter never exceeding $100 \mathrm{mV}$ ) straight lines were obtained, the slope of which corresponded to the electronic conductance, $C_{\mathrm{e}}$. The ratio between $C_{\mathrm{e}}$ and the total conductance, $C_{\mathrm{t}}$, obtained in a.c. with a $10^{4} \mathrm{rad} / \mathrm{sec}$ frequency, gave the electronic transport number, $t_{\mathrm{e}}$, with a good approximation.

Figure 2 shows some typical results from which it is apparent that $t_{\mathrm{e}}=0.6 \pm 0.1$ for $\beta$-TII at $100 \div 185^{\circ} \mathrm{C}$, whilst for the $\alpha$-phase $t_{\mathrm{e}} \approx 0$ at $200{ }^{\circ} \mathrm{C}$.

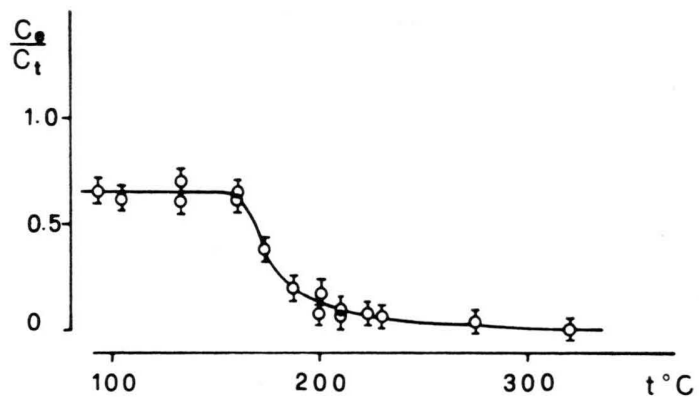

Fig. 2. $\beta-\alpha$ transition of $\mathrm{Tl}$ I detected by electronic conductance determinations; the ratio, $C_{\mathrm{e}} / C_{\mathrm{t}}$, between electronic and total conductance gives the electronic transport number.

As for ionic transport numbers in TII, Table 1 reports the results of several determinations in $\alpha$-TII in the temperature range $206-255^{\circ} \mathrm{C}$. Measurements at higher temperatures were not reliable owing to the sublimation of TII.

The $t_{\mathrm{Tl}}$ values were calculated on the basis of $\Delta P_{\text {cathodic }}$ and $\Delta P_{\text {anodic }}$, which refer to the total weight variation of the compartement, i.e., electrode + adjacent pellet.

The data fluctuation and the narrow temperature range did not allow to recognize a particular dependence on temperature.

Taking into account the experimental difficulties of Tubandt transport number determinations on $\mathrm{Tl}$ halides ${ }^{11}$, the given values of $t_{\mathrm{Tl}}$ must be considered correct within $\pm 15 \%$.

Tab. 1. Tubandt transport numbers determinations on the cell $+\mathbf{A g} / \mathrm{Tl} / \mathrm{Tl} \mathrm{I} / \mathrm{Tl} \mathrm{I} / \mathrm{Ag}-. \Delta P$ values are referred to 10 coulombs flowed through the cell.

\begin{tabular}{|c|c|c|c|c|c|}
\hline Compartement & $T=206^{\circ} \mathrm{C}$ & $T=212{ }^{\circ} \mathrm{C}$ & $T=224{ }^{\circ} \mathrm{C}$ & $T=225^{\circ} \mathrm{C}$ & $T=255^{\circ} \mathrm{C}$ \\
\hline $\begin{array}{l}\Delta P_{\text {anodic }} \\
\Delta P_{\text {eathodic }}\end{array}$ & $\begin{array}{l}+4.05 \mathrm{mg} \\
-4.21 \mathrm{mg}\end{array}$ & $\begin{array}{l}+3.86 \mathrm{mg} \\
-3.51 \mathrm{mg}\end{array}$ & $\begin{array}{l}+2.61 \mathrm{mg} \\
-3.44 \mathrm{mg}\end{array}$ & $\begin{array}{l}+2.89 \mathrm{mg} \\
-2.65 \mathrm{mg}\end{array}$ & $\begin{array}{c}+3.40 \mathrm{mg} \\
-2.10 \mathrm{mg}\end{array}$ \\
\hline $\begin{array}{l}t_{\mathrm{Tl}} \\
\text { from anode } \\
t_{\mathrm{T} l}\end{array}$ & $26.5 \%$ & $27.1 \%$ & $30.7 \%$ & $29.9 \%$ & $28.4 \%$ \\
\hline from cathode & $26.0 \%$ & $28.1 \%$ & $28.3 \%$ & $30.6 \%$ & $32.2 \%$ \\
\hline
\end{tabular}


The table figures show that in $\alpha$-TII, as well as in $\mathrm{TlCl}$ and $\mathrm{TlBr}$, the principal carrier is the anion, although the $\mathrm{Tl}^{+}$contribution to the ionic conductivity, amounting to about $30 \%$, is not negligible.

The ionic transport numbers in $\beta$-TII, which should attain about $40 \%$, could not be determined because the high electrical resistance would require too long periods or too high voltages for an electrolytic process to become able to give detectable weight variations.

\section{The AgI-Tll System}

The phase diagram, as determined by Berg and Lepeshkov ${ }^{12}$, was substantially confirmed.

In Fig. $3 \log \sigma$ vs $1 / T$ is plotted for TII, AgI and the peculiar compositions $X_{\mathrm{AgI}}=0.33$ and $X_{\mathrm{AgI}}$ $=0.5$, corresponding to the two known intermediate compounds ${ }^{13,14}$.

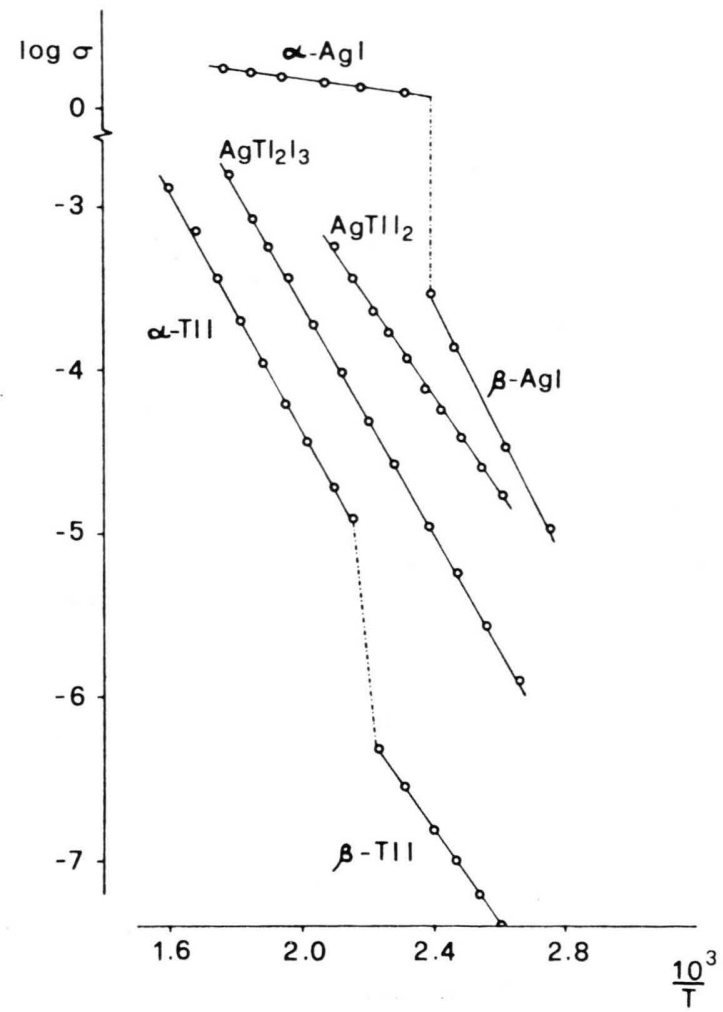

Fig. 3. Arrhenius plots of the specific conductivity for Tl I, $\mathrm{AgTl}_{2} \mathrm{I}_{3}, \mathrm{AgTl}_{2}$ and $\mathrm{AgI}$.

The latter were prepared by: either 150 hours annealing of proper powder mixtures at $190{ }^{\circ} \mathrm{C}$; or coprecipitation with excess KI from aqueous solutions containing $\mathrm{AgNO}_{3}$ and $\mathrm{TINO}_{3}$ in the ratios
$1: 2$ and $1: 1$; or slow cooling of the melts and some hours rest at the freezing temperatures.

As for the first procedure, $\mathrm{X}$ rays analysis showed that the annealing time at $190{ }^{\circ} \mathrm{C}$ was critical for the complete formation of $\mathrm{AgTl}_{2} \mathrm{I}_{3}$ and $\mathrm{AgTlI}_{2}$ : e. g., only after a previous 150 hours annealing of the $X_{\mathrm{AgI}}=0.5$ powder mixture the peaks of $\mathrm{AgI}$ and $\mathrm{AgTl}_{2} \mathrm{I}_{3}$ vanished and a diffraction pattern was obtained showing only the $\mathrm{AgTlI}_{2}$ peaks.

The not reported plots $\log \sigma$ vs $1 / T$ showed a conductivity knee respectively at $145{ }^{\circ} \mathrm{C}(\mathrm{AgI} \beta \rightarrow u$ transition) for $\mathrm{AgTlI}_{2}$ and at $185^{\circ} \mathrm{C}$ (TII $\beta \rightarrow \alpha$ transition) for $\mathrm{AgTl}_{2} \mathrm{I}_{3}$, when the compounds were obtained by the first and second procedure, while such knees were completely absent (see Fig. 3) when the third procedure was followed.

In the latter case the intermediate compounds can be considered sufficiently pure for transport phenomena investigations.

Transport number determinations with the Tubandt method showed that in both compounds the silver ions must be considered as the only carriers; the results at $160{ }^{\circ} \mathrm{C}$ and $162^{\circ} \mathrm{C}$ are reported in Table 2.

Tab. 2. Tubandt transport number determinations on the cells $\mathrm{Ag} / \mathrm{AgI} / \mathrm{AgTl}_{2} \mathrm{I}_{3} / \mathrm{AgI} / \mathrm{Ag}$ and $\mathrm{Ag} / \mathrm{AgI} / \mathrm{AgTl} \mathrm{I}_{2} / \mathrm{AgI} / \mathrm{Ag}$.

\begin{tabular}{lcc}
\hline Compartement & $\mathrm{AgTl}_{2} \mathrm{I}_{3} \mathrm{~T}=160^{\circ} \mathrm{C}$ & $\mathrm{AgTlI}_{2} T=162{ }^{\circ} \mathrm{C}$ \\
\hline$\Delta P_{\text {anodic }}$ & $-21.20 \mathrm{mg}$ & $-11.80 \mathrm{mg}$ \\
central pellet & $+0.60 \mathrm{mg}$ & $+0.65 \mathrm{mg}$ \\
$\Delta P_{\text {cathodic }}$ & $+19.05 \mathrm{mg}$ & $+12.25 \mathrm{mg}$ \\
flowed charge & $18 \mathrm{coul}$. & $10.8 \mathrm{coul}$. \\
weigth variation & $20.12 \mathrm{mg}$ & $12.07 \mathrm{mg}$ \\
in coulometer & & \\
silver electr. & & 1 \\
\multicolumn{1}{c}{$t_{\mathrm{Ag}}$} & 1 & 1 \\
\hline
\end{tabular}

\section{Final Remarks}

The experimental results lead to the conclusion that the type of conductivity markedly varies passing from TII to $\mathrm{AgTl}_{2} \mathrm{I}_{3}, \mathrm{AgTlI}_{2}$ and $\mathrm{AgI}$ : the electronic conductivity, significant in $\beta$-TII, vanishes in the intermediate compounds as well as in $\mathrm{AgI}$; $\alpha$-TII shows a predominant anionic conductivity which is totally absent in the other compounds; the ionic transport number of $\mathrm{Tl}^{+}$falls to zero in $\mathrm{AgTl}_{2} \mathrm{I}_{3}$ and $\mathrm{AgTlI}_{2}$ where the silver ions are the only carriers. 
Furthermore, the latter compounds show a much lower conductivity than other silver ion conducting poliiodides.

This is consistent with the crystal structure of $\mathrm{AgTlI}_{2}$ and $\mathrm{AgTl}_{2} \mathrm{I}_{3}{ }^{14}$ which is by no means an "open lattice" structure, as it seems to be required for a good ionic conductivity in solids.

The low conductivity of these salts can also be related to the theories for the crystal bond ionicity by Phillips and Van Vechten ${ }^{15,16}$ and by Tubbs ${ }^{17}$, which predict in $\mathrm{M}^{n} \mathrm{X}^{8-n}$ salts a 4 -fold or a 6 -fold coordination for the cations according to the fact that ionicities of the crystal bonds lie below or

1 C. Tubandt and E. Lorenz, Z. Phys. Chem. 87, 513543 [1914].

2 R. J. Friauf, J. Phys. Chem. Solids 18, 203 [1961]. - R. J. Friauf, Z. Naturforsch. 26 a, 1210 [1971].

3 W. A. Bassett and T. Takahashi, Am. Mineral. 50, 1576 [1965].

4 H. Hoshino and M. Shimoji, J. Phys. Chem. Solids 33, 2303 [1972].

5 G. A. Samara, L. C. Walters, and D. A. Northrop, J. Phys. Chem. Solids 28, 1875 [1967]

6 M. H. Hebb, J. Chem. Phys. 20, 185 [1952].

7 I. Yokota, J. Phys. Soc. Japan 16, 2213 [1961].

8 R. W. Vest and N. M. Tallan, J. Appl. Phys. 36, 543 [1965].

9 T. E. Phipps and E. G. Partridge, J. Am. Chem. Soc. 51, 1331 [1929]. above a critical value $(0.785$ according to Phillips or 0.51 according to Tubbs). For both theories AgI performs ionicity just below and quite close to the critical value, so that on adding to $\mathrm{AgI}$ a highly ionic salt such as $\mathrm{RbI}, \mathrm{NH}_{4} \mathrm{I}$ or $\mathrm{KI}$ one obtains compounds where either coordination is possible; this results in many energetically equivalent positions for a silver ion and consequently in a very good conductivity ${ }^{18}$.

This may not occur when TII (whose ionicity is very low) is added to $\mathrm{AgI}$.

The authors are indebted to Prof. M. Rolla and Prof. C. Sinistri for useful critical discussion.

10 Z. Morlin, Phys. Stat. Sol. (a) 8, 565 [1971].

11 P. Hermann, Z. Phys. Chem. 227, 338 [1964].

12 Berg and Lepeshkov, Izvest. Sekt. Fiz. Khim. Anal. Inst. Obshchei Neorg. Khim. 15, 144 [1947]; Chem. Abs. 44, 7134 [1950].

13 H. Hirsh, J. Chem. Soc. 246, 1318 [1963].

14 P. Messien, Bull. Soc. Roy. Sci. Liège 38, 490 [1969].

15 J. A. Van Vechten, Phys. Rev. 182, 891 [1969].

16 J. C. Phillips and J. A. Van Vechten, Phys. Rev. Lett. 22, 705 [1969]; J. C. Phillips, Rev. Modern Phys. 42, 317 [1970].

17 M. R. Tubbs, Phys. Stat. Sol. 41, K 61 [1970].

18 D. Butherus, J. C. Phillips, and B. Scrosati, Fall Meeting of the Electrochem. Soc., Miami Beach, Florida (USA), Oct. 1972, Abstract n. 11 . 